alimentaire, concourent à rendre la production du lait, non seulement plus ou moins coûteuse et plus ou moins bienfaisante pour le bétail, mais encore à modifier profondément la flore bactérienne du lait (d'après les recherches du Professeur GoRini dans le lait " disgenesico ") en obtenant des effets remarquables par rapport au traitement du lait et aux résultats des produits fromagers.

On peut donc déduire la nécessité d'ordre spéculatif et d'ordre pratique d'orienter les recherches vers le champ d'alimentation des vaches laitières, de façon à pouvoir identifier l'action fromagère ou anti-fromagère des aliments les plus communs, afin d'établir leur association convenable zooéconomique, et pour exploiter et équilibrer les actions antagonistes et compensatrices.

Pour finir, nous dirons que pour ce qui concerne les résultats de l'expérience susdite, ainsi que leur exposition et leur discussion, on est contraint pour l'instant de les ajourner. Ces résultats seront recueillis dans un futur article, que nous nous réservons de préparer dès qu'il nous sera possible.

Pourtant on a commencé en 1936 d'autres expériences sur l'alimentation du bétail et sur le contrôle fromager, expériences quî sont actuellement en cours et qui comprennent un champ d'action beaucoup plus vaste que le précédent.

Pavie, 5 mars 1936.

\title{
QUELQUES OBSERVATIONS CONCERNANT L'ACTION DE LA SOLUTION DE CHLORURE DE CALCIUIM POUR RÉFRACTOMÉTRIE SUR LES LAITS ADDITIONNÉS DE CARBONATES ALCALINS
}

\author{
par \\ A. TAPERNOUX \\ Professeur de Chimie \\ à l'Ecole Vétérinaire de Lyon
}

Une très longue pratique de la réfractométrie appliquée au lait, en utilisant la méthode de préparation du sérum chlorocalcique, selon Ackermann [1, 2], m'a permis de faire l'observation suivante: les laits non altérés par la fermentation lactique ou par un trouble de la sécrétion, mais qui ont été additionnés de carbonates ou de bicarbonates alcalins, coagulent mal ou ne coagulent pas du tout au cours des manipulations employées pour préparer le sérum. Si la dose de sel alcalin ajoutée au lait est faible, la coagulation se produit et la synérèse laisse sourdre un sérum trouble; si la dose de sel alcalin est plus forte, le mélange lait + solution de chlorure de calcium 
porté au bain-marie bouillant mousse considérablement et ne coagule pas.

Cette observation faite à maintes reprises m'a conduit à réaliser quelques expériences que ce travail a pour but de rapporter; je n'en tire pour l'instant aucune conclusion définitive et j'insiste sur ce point, aucune méthode précise de recherche des conservateurs alcalins ajoutés au lait ; tout au plus peut-elle avoir quelque utilité dans le dépistage rapide d'une fraude encore trop répandue et qui consiste à additionner le lait de carbonates ou de bicarbonates alcalins, pour prévenir les effets d'une altération rapide ou pour masquer les résultats du développement brutal de l'acidification.

Dans sa thèse de doctorat vétérinaire, Commeny [3] a montré quels étaient les résultats déplorables du point de vue de l'Hygiène de la pratique du biearbonatage ou du "poudrage" - suivant l'expression des fraudeurs - appliquée au lait de consommation en nature pendant les mois de chaleur; je n'y reviendrai pas.

Il est utile d'indiquer en quelques mots la technique suivie pour préparer le sérum chlorocalcique destiné à la réfractométrie, technique du reste bien connue et que je me bornerai à rappeler. On prépare tout d'abord une solution que je désignerai par A en dissolvant 200 grammes de chlorure de calcium fondu dans une quantité suffisante d'eau distillée pour obtenir 1 litre de solution ; la solution doit être ajustée de telle sorte que sa densité à $15^{\circ}$ soit de 1,1375 et que diluée au $1 / 10^{\mathrm{e}}$ ( 1 volume pour 9 volumes d'eau distillée) elle présente au réfractomètre à immersion de Zeiss un chiffre réfractométrique de 26 à $17^{\circ} 5$, ce qui correspond à un indice $\mathrm{n}=1.33743$.

La manipulation de préparation du sérum consiste à introduire dans l'éprouvette spéciale de 230 millimètres de long et de 20 millimètres de diamètre (à la rigueur un gros tube à essai de 200/20e peut suffire) $30 \mathrm{~cm}^{3}$ de lait à examiner et $0 \mathrm{~cm}^{3} 25$ de solution A. On agite vigoureusement et on obture l'éprouvette avec un bouchon de caoutchouc muni d'un tube réfrigérant de 260 millimètres de long et de 7 millimètres de diamètre, puis on porte l'éprouvette dans un bain-marie dont l'eau a été préalablement portée à l'ébullition et on l'y laisse 15 minutes. On la retire ensuite et on la refroidit immédiatement dans une cuve d'eau courante.

Normalement, pendant le séjour du tube au bain-marie, bouillant, le mélange lait + solution $\mathrm{A}$ a coagulé et un sérum limpide a exsudé en quantité suffisante pour être décanté dans l'un des godets du réfractomètre et pour être soumis à l'épreuve de cet instrument. Dans certains cas cependant, la coagulation est défectueuse ou ne se produit pas. 
Parmi les causes qui déterminent cette anomalie de la coagulation, l'observation m'a montré qu'il fallait faire une place à l'addition préalable au lait de carbonates ou de bicarbonates alcalins. Mon ami regretté PANCHAUD [4] a insisté sur les défectuosités observées dans la coagulation des laits qui ont subi la fermentation lactique et avec lesquels on obtient un sérum trouble dont l'indice de réfraction est anormalement élevé. C'est la raison pour laquelle, dans la pratique courante, je me borne à déterminer la réfraction des laits dont l'acidité est inférieure à $25^{\circ}$ Dornic, ce qui réduit un peu l'intérêt de cette méthode de détection rapide du mouillage. On peut encore obtenir des coagulations défectueuses avec les laits de mammite ou de rétention (1), mais en dehors de ces cas, et alors que le lait examiné apparaît normal, la coagulation défectueuse peut être la conséquence du " poudrage " que j'ai signalé.

A la suite des observations qui m'ont conduit sur cette voie, j'ai réalisé quelques expériences que je vais maintenant décrire.

Le lait utilisé pour toutes ces expériences était un lait de mélange pasteurisé à la température de $85^{\circ}$ dans un pasteurisateur à plaques (système Ahlborn). Son acidité Dornic était de $18^{\circ}$.

10 J'ai d'abord déterminé la quantité limite de solution A qui coagule à l'ébullition $30 \mathrm{~cm}^{3}$ de lait ; voici les résultats :

Volume de solution A ajouté à $30 \mathrm{~cm}^{3}$ de lait

$$
\begin{aligned}
& 0 \mathrm{~cm}^{3} 10 \\
& 0 \mathrm{~cm}^{3} 15 \\
& 0 \mathrm{~cm}^{3} 20 \\
& 0 \mathrm{~cm}^{3} 25 \\
& 0 \mathrm{~cm}^{3} 50
\end{aligned}
$$

Résultat

Pas de coagulation.

Coagulation en flocons.

Sérum très trouble.

Coagulation en masse.

Sérum clair.

Id.

Id.

Par conséquent, la dose limite pour obtenir une coagulation est, dans l'expérience effectuée, de $0 \mathrm{~cm}^{3} 15$ de solution A.

$2^{\circ}$ Le même lait a été ensuite additionné de doses variables de bicarbonate de sodium. Quatre échantillons contenant respectivement $0 \mathrm{gr}$. 25, $0 \mathrm{gr}$. 50, 1 gr. et $2 \mathrm{gr}$. par litre, ont été traités par des volumes variables de solution $A$. Les résultats sont consignés dans le tableau suivant.

On note en même temps qu'une mousse abondante se développe à la surface du lait au cours de l'ébullition et il semble qu'elle est plus intense lorsque la dose de bicarbonate est plus élevée.

Il est à remarquer au cours de cette série d'essais que la dose de $0 \mathrm{~cm}^{3} 50$ de solution A est peu intéressante puisque la coagulation

(1) Voir les observations de Metzger, Fuchs et Jesser cités par Lataix. Le Lait, 1929 , p. 487. 


\begin{tabular}{|c|c|c|c|c|c|}
\hline \multicolumn{2}{|c|}{$\mathrm{CO}^{3} \mathrm{NaH} 0 / 00$} & 0 gr. 25 & 0 gr. 50 & $1 \mathrm{gr}$. & $2 \mathrm{gr}$. \\
\hline$\ddot{+}$ & $\begin{array}{l}0 \mathrm{~cm}^{3} 20 \\
\text { sol. A }\end{array}$ & $\begin{array}{c}\text { Coagule } \\
\text { sérum trouble }\end{array}$ & $\begin{array}{c}\text { Ne coagule } \\
\text { pas }\end{array}$ & $\begin{array}{l}\text { Ne coagule } \\
\text { pas }\end{array}$ & $\begin{array}{c}\text { Ne coagule } \\
\text { pas }\end{array}$ \\
\hline$\frac{\frac{\pi}{7}}{\frac{0}{2}}$ & $\begin{array}{l}0 \mathrm{~cm}^{3} 25 \\
\text { sol. A }\end{array}$ & $\begin{array}{l}\text { Coagule } \\
\text { sérum clair }\end{array}$ & $\begin{array}{l}\text { Coagule } \\
\text { sérum trouble }\end{array}$ & $\begin{array}{c}\text { Ne coagule } \\
\text { pas }\end{array}$ & $\begin{array}{c}\text { Ne eoagule } \\
\text { pas }\end{array}$ \\
\hline क् & $\begin{array}{l}0 \mathrm{~cm}^{3} 50 \\
\text { sol. A }\end{array}$ & $\begin{array}{l}\text { Coagule } \\
\text { sérum clair }\end{array}$ & $\begin{array}{l}\text { Coagule } \\
\text { sérum clair }\end{array}$ & $\begin{array}{l}\text { Coagule } \\
\text { sérum elair }\end{array}$ & $\begin{array}{c}\text { Coagule } \\
\text { sérum trouble }\end{array}$ \\
\hline
\end{tabular}

a lieu pour tous les échantillons de lait; d'ailleurs, cette dose est trop élevée pour la préparation du sérum; c'est pourquoi nous l'avons abandonnée pour les expériences suivantes.

$3^{\circ}$ Le même lait a été ensuite additionné de carbonate de sodium anhydre. Quatre échantillons ont été préparés dans lesquels j'ai recherché une dose correspondant aux quatre doses de bicarbonate de sodium employées dans l'expérience $n^{\circ} 2$. Or, les solutions de bicarbonate soumises à l'ébullition se transforment, en donnant avec dégagement de $\mathrm{CO}^{2}$, des solutions de carbonate, d'après la réaction :

$$
2 \mathrm{CO}^{3} \mathrm{NaH}=\mathrm{CO}^{3} \mathrm{Na}^{2}+\mathrm{CO}^{2}+\mathrm{H}^{2} \mathrm{O}
$$

On peut penser qu'une réaction analogue se produit lorsqu'on porte à l'ébullition du lait additionné de bicarbonate de sodium; cette opinion est étayée par la détermination de l'acidité de titration des laits au moyen de la soude N/9 (Dornic) et de la phtaléine du phénol comme indicateur. Les solutions de bicarbonate de soude sont neutres à la phtaléine du phénol ou très légèrement alcalines alors que les solutions de carbonate de sodium se eomportent comme des bases vis-à-vis de cet indicateur. Un lait additionné de bicarbonate présente done une acidité inchangée ou presque lorsque la détermination sera faite avant l'ébullition.

Au contraire, ce même lait préalablement soumis à l'ébullition présentera une acidité diminuée proportionnellement à la dose de bicarbonate ajoutée, par suite de la transformation du bicarbonate en carbonate neutre. HINARD avait tiré de cette observation une méthode de recherche du bicarbonate de soude dans le lait [5].

L'équation de transformation montre que 168 grammes de $\mathrm{CO}^{3} \mathrm{NaH}$ correspondent à 106 grammes de $\mathrm{CO}^{3} \mathrm{Na}^{2}$; done les doses qui devront être employées pour les quatre échantillons seront approximativement :

0 gr. 16

0 gr. 32

0 gr. 64

1 gr. 28 
Mes quatre échantillons de lait ainsi préparés sont traités par la solution $\mathrm{A}$, comme ceux de l'expérience $\mathrm{n}^{\circ} 2$; les résultats obtenus sont consignés dans le tableau qui suit :

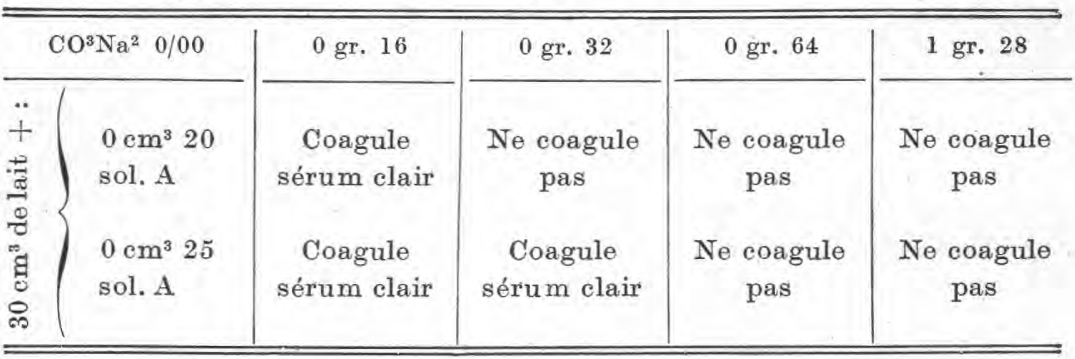

Les résultats de cette expérience concordent avec ceux de l'expérience $\mathrm{n}^{\circ} 2$ en ce qui concerne la coagulation; l'aspect du sérum mis à part, les deux séries d'essais sont exactement superposables.

$4^{\circ}$ On peut utiliser pour préparer les échantillons de lait d'autres carbonates que ceux de sodium; j'ai expérimenté le carbonate de lithium $\mathrm{CO}^{3} \mathrm{Li}^{2}$ etle sesqui-carbonate d'ammonium $\left(\mathrm{CO}^{3}\right)^{3}\left(\mathrm{NH}^{4}\right)^{4} \mathrm{H}^{2}$. Les doses de chacun de ces sels ont été calculées en tenant compte qu'une molécule gramme de $\mathrm{CO}^{3} \mathrm{Na}^{2}$ (106 grammes) équivaut à une molécule-gramme de $\mathrm{CO}^{3} \mathrm{Li}^{2}$ (74 grammes) ou à $1 / 3$ de moléculegramme de sesqui-carbonate d'ammonium (84 gr. 66).

Les deux tableaux présentés ci-dessous résument pour chaque sel les résultats expérimentaux :

\begin{tabular}{|c|c|c|c|c|c|}
\hline \multicolumn{2}{|c|}{$\mathrm{CO}^{3} \mathrm{Li}^{2} 0 / 00$} & 0 gr. 11 & 0 gr. 22 & 0 gr. 44 & 0 gr. 88 \\
\hline 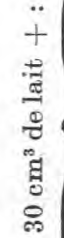 & $\begin{array}{l}0 \mathrm{~cm}^{3} 20 \\
\text { sol. A } \\
0 \mathrm{~cm}^{3} 25 \\
\text { sol. A }\end{array}$ & $\begin{array}{l}\text { Coagule } \\
\text { sérum clair } \\
\text { Coagule } \\
\text { sérum clair }\end{array}$ & $\begin{array}{c}\text { Ne coagule } \\
\text { pas } \\
\text { Flocule par- } \\
\text { tiellement } \\
\text { Coagule } \\
\text { sérum clair }\end{array}$ & $\begin{array}{c}\text { Ne coagule } \\
\text { pas } \\
\text { Ne coagule } \\
\text { pas }\end{array}$ & $\begin{array}{c}\text { Ne coagule } \\
\text { pas } \\
\text { Ne coagule } \\
\text { pas }\end{array}$ \\
\hline$\left(\mathrm{CO}^{3}\right.$ & $\left.\mathrm{NH}^{4}\right)^{4} \mathrm{H}^{2} 0 / 00$ & 0 gr. 13 & 0 gr. 26 & 0 gr. 52 & 1 gr. 04 \\
\hline 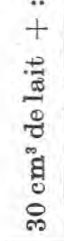 & $\begin{array}{l}0 \mathrm{~cm}^{3} 25 \\
\text { sol. A }\end{array}$ & $\begin{array}{c}\text { Coagule } \\
\text { sérum clair } \\
\text { Coagule } \\
\text { sérum clair }\end{array}$ & $\begin{array}{c}\text { Coagule } \\
\text { sérum trouble }\end{array}$ & $\begin{array}{c}\text { Ne coagule } \\
\text { pas } \\
\text { Coagule } \\
\text { sérum trouble }\end{array}$ & $\begin{array}{c}\text { Ne coagule } \\
\text { pas } \\
\text { Ne coagule } \\
\text { pas }\end{array}$ \\
\hline
\end{tabular}


Les résultats fournis par les laits additionnés de carbonate de lithium sont superposables à ceux donnés par les laits additionnés de carbonate et de bicarbonate de sodium. Les échantillons additionnés de sesqui-carbonate d'ammoniaque présentent une légère différence à la coagulation. J'ai adopté pour ce sel la formule donnée par le Codex, par le traité de Pharmacie de Crolas et Moreau, mais il est bien évident qu'il peut se faire que l'échantillon sur lequel j'ai expérimenté ne corresponde pas très exactement à cette formule, Quoi qu'il en soit, l'allure générale du phénomèné est la même.

Pour expliquer les anomalies présentées par les laits additionnés de carbonates alcalins, on peut penser que ces sels neutralisent le chlorure de calcium suivant l'équation du type :

$$
\mathrm{CO}^{3} \mathrm{Na}^{2}+\mathrm{CaCl}^{2}=\mathrm{CO}^{3} \mathrm{Ca}+2 \mathrm{NaCl}
$$

Cette équation montre qu'à 106 grammes de carbonate de sodium correspondent 111 grammes de chlorure de calcium anhydre ; or l'addition de $0 \mathrm{~cm}^{3} 20$ de solution $\mathrm{A}$ à $30 \mathrm{~cm}^{3}$ de lait équivaut à l'addition de $1 \mathrm{gr}$. 33 de ehlorure de calcium à $1.000 \mathrm{em}^{3}$ de lait. D'autre part, l'addition de $0 \mathrm{~cm}^{3} 25$ de solution A à $30 \mathrm{~cm}^{3}$ de lait équivaut à l'addition de $1 \mathrm{gr}$. 66 de chlorure de calcium à $1.000 \mathrm{~cm}^{3}$ de lait. L'équation écrite plus haut montre qu'à $1 \mathrm{gr}$. 33 de chlorure de calcium correspond 1 gr. 27 de carbonate de sodium et qu'à 1 gr. 66 de chlorure de calcium correspond 1 gr. 58 de carbonate de sodium.

Or, dans ma première expérience, j'ai montré qu'il faut au, minimum $0 \mathrm{~cm}^{3} 15$ de solution A pour obtenir un coagulum floconneux limite avec un lait témoin. Donc, en réalité, sur $0 \mathrm{~cm}^{3} 20$, seulement $0 \mathrm{~cm}^{3} 05$ sont utilisés pour neutraliser le carbonate de sodium et sur $0 \mathrm{~cm}^{3} 25$, seulement $0 \mathrm{~cm}^{3} 10$ sont utilisés pour la neutralisation; dans le premier cas, un calcul simple nous montre que $0 \mathrm{~cm}^{3} 05$ de solution $\mathrm{A}$ correspond à $0 \mathrm{gr}$. 32 de carbonate de sodium. Donc, tout lait renfermant moins de 0 gr. 32 de carbonate de sodium doit coaguler et tout lait renfermant plus de $0 \mathrm{gr}, 32$ de carbonate de sodium ne doit pas coaguler par addition de $0 \mathrm{~cm}^{3} 20$ de solution A. C'est ce que l'expérience vérifie.

Dans le deuxième cas, tout lait renfermant moins de 0 gr. 64 de carbonate de sodium doit coaguler, et tout lait renfermant plus de 0 gr. 64 de carbonate de sodium ne doit pas coaguler; e'est également ce que l'expérience vérifie.

Un calcul semblable montrerait qu'il doit exister également pour les autres sels une limite de concentration qui détermine la coagulation ou la non-coagulation de l'échantillon envisagé.

J'ai résumé dans le tableau suivant les quantités des différents sels examinés qui m'ont paru équivalents : 


\begin{tabular}{c|c|c|c|c}
\hline \multicolumn{1}{c|}{ Sels étudiés } & \multicolumn{3}{|c}{ Doses équivalentes p. $1.000 \mathrm{~cm}^{3}$ de lait } \\
\cline { 2 - 4 } $\mathrm{CO}^{2} \mathrm{NaH} \ldots \ldots \ldots$ & 0 gr. 25 & 0 gr. 50 & 1 gr. & 2 gr. \\
$\mathrm{Co}^{3} \mathrm{Na}^{2} \ldots \ldots \ldots$ & 0 gr. 16 & 0 gr. 32 & 0 gr. 64 & 1 gr. 28 \\
$\mathrm{Co}^{3} \mathrm{Li}^{2} \ldots \ldots \ldots \ldots$ & 0 gr. 11 & 0 gr. 22 & 0 gr. 44 & 0 gr. 88 \\
$\left(\mathrm{CO}^{3}\right)^{3}\left(\mathrm{NH}^{4}\right)^{4} \mathrm{H}^{2} \ldots$ & 0 gr. 13 & 0 gr. 26 & 0 gr. 52 & 1 gr. 04 \\
\hline \hline
\end{tabular}

Pour compléter cette série d'expériences, j'ai recherché si l'addition d'un antiseptique modifiait la production du phénomène. J'ai tout d'abord additionné le lait examiné de 1 gramme par litre de bichromate de potassium et j'ai obtenu les résultats consignés dans le tableau ci-dessous :

Laits additionnés de $10 / 00 \mathrm{Cr}^{2} \mathrm{O}^{7} \mathrm{~K}^{2}+0 \mathrm{~cm}^{3} 20$ sol. A

\begin{tabular}{|c|c|c|c|c|c|}
\hline Sel ajouté & Témoin & $1^{\text {re }}$ dos̉e & $2^{e}$ dose & $3^{e}$ dose & $4^{e}$ dose \\
\hline $\mathrm{CO}^{3} \mathrm{NaH} \ldots \ldots$ & $\begin{array}{c}\text { Coagule } \\
\text { sérum clair }\end{array}$ & $\begin{array}{c}\text { Coagule } \\
\text { sérum un } \\
\text { peu trouble }\end{array}$ & $\begin{array}{c}\text { Ne coagule } \\
\text { pas }\end{array}$ & $\begin{array}{c}\text { Ne coagule } \\
\text { pas }\end{array}$ & $\begin{array}{c}\text { Ne eoagule } \\
\text { pas }\end{array}$ \\
\hline $\mathrm{CO}^{3} \mathrm{Na}^{2}$. & $\begin{array}{c}\text { Coagule } \\
\text { sérum clair }\end{array}$ & $\begin{array}{c}\text { Coagule } \\
\text { sérum clair }\end{array}$ & $\begin{array}{c}\text { Ne coagule } \\
\text { pas }\end{array}$ & $\begin{array}{c}\text { Ne coagule } \\
\text { pas }\end{array}$ & $\begin{array}{c}\text { Ne coagule } \\
\text { pas }\end{array}$ \\
\hline $\mathrm{CO}^{3} \mathrm{Li}^{2}$ & $\begin{array}{c}\text { Coagule } \\
\text { sérum clair }\end{array}$ & $\begin{array}{c}\text { Coagule } \\
\text { sérum } \\
\text { trouble }\end{array}$ & $\begin{array}{l}\text { Coagulat. } \\
\text { incomplète } \\
\text { sérum tr. }\end{array}$ & $\begin{array}{c}\text { Ne coagule } \\
\text { pas }\end{array}$ & $\begin{array}{c}\text { Ne coagule } \\
\text { pas }\end{array}$ \\
\hline$\left(\mathrm{CO}^{3}\right)^{3}\left(\mathrm{NH}^{4}\right)^{4} \mathrm{H}^{2}$. & $\begin{array}{c}\text { Coagule } \\
\text { sérum clair }\end{array}$ & $\begin{array}{c}\text { Coagule } \\
\text { sérum clair }\end{array}$ & $\begin{array}{c}\text { Coagule } \\
\text { sér. troub. }\end{array}$ & $\begin{array}{c}\text { Ne coagule } \\
\text { pas }\end{array}$ & $\begin{array}{c}\text { Ne coagule } \\
\text { pas }\end{array}$ \\
\hline
\end{tabular}

L'expérience montre que les résultats sont approximativement les mêmes lorsque le lait a été additionné de bichromate de potassium. Je n'ai pas pour l'instant examiné les laits profondément altérés, comme ceux que l'on rencontre au cours des expertises judiciaires, mais je me propose de compléter ces expériences par l'étude de ces échantillons. Toutefois, j'ai remarqué que le développement de la fermentation lactique amenait des modifications dans la coagulation des laits bicarbonatés, sous l'influence du chlorure de calcium. Ces modifications ne sont d'ailleurs pas spéciales aux laits additionnés de conservateurs alcalins. Voici le résumé de deux expériences faites à ce sujet ; 
Lait cru additionné de $\mathrm{CO}^{3} \mathrm{NaH}+0 \mathrm{~cm}^{3} 20$ de solution $\mathrm{A}$

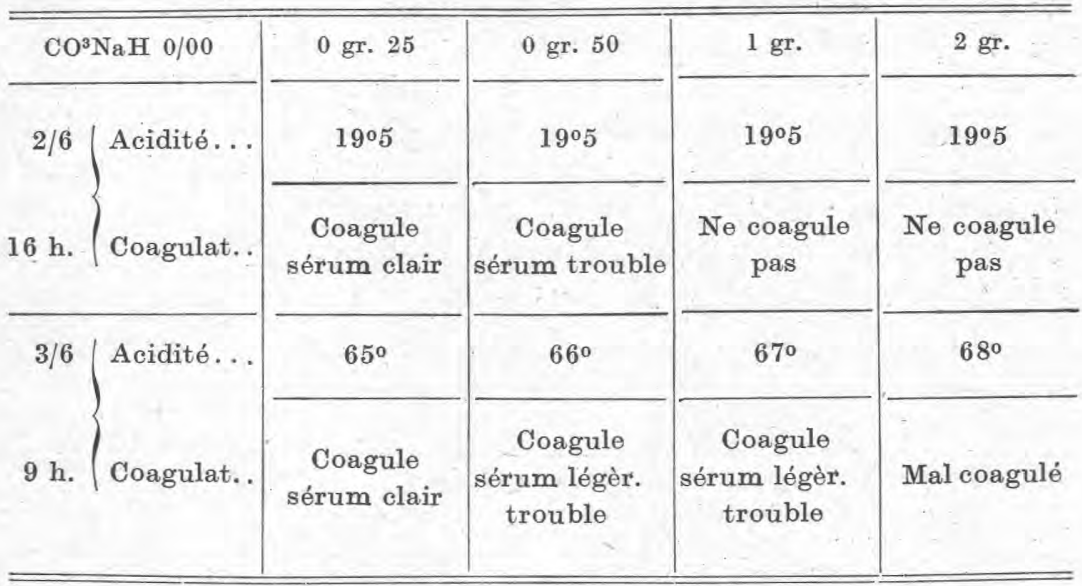

Lait pasteurisé additionné de $\mathrm{CO}^{3} \mathrm{NaH}+0 \mathrm{~cm}^{3} 20 \mathrm{sol}$. A

\begin{tabular}{|c|c|c|c|c|}
\hline $\mathrm{CO}^{3} \mathrm{NaH} 0 / 00$ & 0 gr. 25 & 0 gr. 50 & $1 \mathrm{gr}$. & 2 gr. \\
\hline 2/6 (Acidité... & $17^{\circ}$ & $17^{\circ}$ & $17^{\circ}$ & $17^{\circ}$ \\
\hline $16 \mathrm{~h}$. Coagulat. & $\begin{array}{c}\text { Coagule } \\
\text { sérum clair }\end{array}$ & $\begin{array}{c}\text { Coagule } \\
\text { sérum trouble }\end{array}$ & $\begin{array}{c}\text { Ne coagule } \\
\text { pas }\end{array}$ & $\begin{array}{c}\text { Ne coagule } \\
\text { pas }\end{array}$ \\
\hline $3 / 6$ (Acidité... & $21^{\circ}$ & $27^{\circ}$ & $31^{\circ}$ & $30^{\circ}$ \\
\hline $9 \mathrm{~h}$. ( Coagulat.. & $\begin{array}{c}\text { Coagule } \\
\text { sér. tr. trouble }\end{array}$ & $\begin{array}{c}\text { Coagule } \\
\text { sér. tr. troub. }\end{array}$ & $\begin{array}{c}\text { Coagule } \\
\text { sér. tr. trouble }\end{array}$ & $\begin{array}{c}\text { Coagule } \\
\text { sér. tr. troubl. }\end{array}$ \\
\hline $3 / 6$ (Acidité... & $39^{\circ}$ & $65^{\circ}$ & $75^{\circ}$ & $70^{\circ}$ \\
\hline $15 \mathrm{~h}$. (Coagulat. & $\begin{array}{c}\text { Coagule } \\
\text { sérum trouble }\end{array}$ & Précipite & Précipite & Précipite \\
\hline
\end{tabular}

Comme on le voit, les laits acides coagulent par addition de chlorure de calcium à des doses qui ne provoquent pas la coagulation lorsque le lait n'a pas fermenté. Il est vrai que ces laits coaguleraient par la chaleur même sans addition de $\mathrm{CaCl}^{2}$ puisque les laits dont l'acidité est supérieure à $25^{\circ}$ Dornic coagulent à l'ébullition. Il était néanmoins intéressant de réaliser l'expérience avee des laits additionnés de conservateurs alcalins. Le deuxième tableau concernant 
le lait pasteurisé permet de se rendre compte de l'effet néfaste du bicarbonate : la fermentation lactique est d'autant plus accélérée que la dose de bicarbonate ajoutée est plus grande.

$J$ 'ai, en outre, examiné si l'addition de $2 \mathrm{~cm}^{3}$ de formol commercial par litre de lait modifiait sensiblement la coagulabilité des laits alcalinisés. Je n’ai expérimenté que sur des laits additionnés de bicarbonate de sodium et le tableau suivant donne le résultat obtenu, qui est à peu près conforme à celui fourni par les laits non additionnés de cet antiseptique :

Laits additionnés de $2 \mathrm{~cm}^{3}$ de formol commercial par litre $+0 \mathrm{~cm}^{3} 20 \mathrm{sol}$. A

\begin{tabular}{c|c|c|c|c|c}
\hline \hline Sel ajouté & Témoin & $1^{\text {re } \text { dose }}$ & $2^{\mathrm{e} \text { dose }}$ & $3^{\mathrm{e} \text { dose }}$ & $4^{\mathrm{o} \text { dose }}$ \\
\cline { 2 - 4 } & $\begin{array}{c}\text { Coagule } \\
\text { Córum clair }\end{array}$ & $\begin{array}{c}\text { Coagulat. } \\
\text { incomplète }\end{array}$ & $\begin{array}{c}\text { Ne coagule } \\
\text { pas }\end{array}$ & $\begin{array}{c}\text { Ne coagule } \\
\text { pas }\end{array}$ & $\begin{array}{c}\text { Ne coagule } \\
\text { pas }\end{array}$ \\
\hline \hline
\end{tabular}

En résumé, les expériences précédentes montrent que très souvent au cours de la détermination de l'indice de réfraction du sérum chlorocalcique du lait, on rencontre des laits qui coagulent mal, et que parmi ceux-ci, il faut ranger ceux qui ont été additionnés de substances conservatrices alcalines et en particulier de carbonates et bicarbonates alcalins.

Sans vouloir, comme j'ai insisté au début de cet article, en tirer immédiatement une méthode de recherche des conservateurs alcalins dans le lait, j'ai pensé qu'il était intéressant de publier ces observations, et l'interprétation que j'ai cru devoir leur donner, pour permettre à ceux qui, comme moi, voudraient compléter cette étude d'apporter leur contribution en la matière.

\section{BIBLIOGRAPHIE}

[1] C. Valencien et L. Panchaud. Triage rapide des laits anormaux par la réfractométrie, la catalasimétrie et l'essai à l'alcool alizarine. Le Lait, 1923, p. 529.

[2] A. Lataix. Contribution à l'étude de la réfractométrie des lactosérums. Le Lait, 1929, p. 483 et suiv.

[3] Commeny. L'addition de bicarbonate de sodium au lait. Thèse Doct. vétérinaire, Lyon 1929.

[4] L. PANChAUD. Sur l'augmentation anormale de l'indice de réfraction du sérum chlorocalcique des laits aigris. Le Lait, 1925, p. 777.

[5] G. Hinard. Analyse des Laits. Encyclopédie des Aide-mémoire. Gauthier-Villars et Masson, éditeurs. 